\title{
Integrating Orbitofrontal Cortex into Prefrontal Theory: Common Processing Themes across Species and Subdivisions
}

\author{
Geoffrey Schoenbaum ${ }^{1}$ and Barry Setlow \\ Department of Psychology, Johns Hopkins University, Baltimore, Maryland 21218, USA
}

\begin{abstract}
Currently, many theories highlight either representational memory or rule representation as the hallmark of prefrontal function. Neurophysiological findings in the primate dorsolateral prefrontal cortex indicate that both features may characterize prefrontal processing. Neurons in the dorsolateral prefrontal cortex encode information in working memory, and this information is represented when relevant to the rules governing performance in a task. In this review, we discuss recent reports of encoding in primate and rat orbitofrontal regions indicating that these features also characterize activity in the orbitofrontal subdivision of the prefrontal cortex. These data indicate that (1) neural activity in the orbitofrontal cortex links the current incentive value of reinforcers to cues, rather than representing the physical features of cues or associated reinforcers; (2) this incentive-based information is represented in the orbitofrontal cortex when it is relevant to the rules guiding performance in a task; and (3) incentive information is also represented in the orbitofrontal cortex in working memory during delays when neither the cues nor reinforcers are present. Therefore, although the orbitofrontal cortex appears to be uniquely specialized to process incentive or motivational information, it may be integrated into a more global framework of prefrontal function characterized by representational encoding of performance-relevant information.
\end{abstract}

There is much debate over the defining functional characteristics of prefrontal subdivisions in the primate (for review, see Wise et al. 1996). Although there are many differences among these proposals, each suggests that the prefrontal cortex is involved in either a representational (working) memory function (Fuster 1985; Goldman-Rakic 1987, 1996; Petrides 1994) or rule representation (Passingham 1993; Dias et al. 1996; Robbins 1996; Wise et al. 1996; Kesner 2000; Wise and Murray 2000). These two functions are not mutually exclusive and, in fact, would likely be complementary. It is therefore not surprising that neurophysiological evidence from the dorsolateral prefrontal cortex seems to support an integration of these functions.

For example, it has long been known that dorsolateral regions are important for performance in delay tasks, and cells in these areas fire selectively to cues across delay periods. These findings have been taken as evidence for a working memory function in these prefrontal subdivisions. More recently, however, it has become clear that information is also represented in these areas according to task demands (Miller 2000). The cue-selective encoding properties of the cells change when the rules guiding performance based on those cues are altered. Working memory repre-

${ }^{1}$ Corresponding author.

E-MAIL schoenbg@jhu.edu; FAX (410) 516-6205.

Article and publication are at www.learnmem.org/cgi/doi/ 10.1101/lm.39901. sentations are therefore not simply maintaining information online, but, rather, seem to reflect the use of that information in guiding choices in the task.

In this review, we discuss neurophysiological results indicating that similar features are characteristic of activity in the orbitofrontal cortex, bringing together data from both primates and rats. Most anatomical connections of this prefrontal subdivision are conserved between these species (Preuss 1995; Ongur and Price 2000). Data obtained across species in comparable tasks show a remarkable degree of convergence (Schoenbaum et al., in press). For example, recording studies have linked firing in orbitofrontal neurons to the innate or acquired significance of stimuli in both rodent and primate species. Neurons respond to specific olfactory or visual cues (Tanabe et al. 1975; Thorpe et al. 1983; Schoenbaum and Eichenbaum 1995a; Yonemori et al. 2000), but they appear to be most responsive to cues that have some biological meaning such as odors of other animals, urine, or feces (Onoda et al. 1984). One recent report indicates that even in the human orbitofrontal cortex, neurons respond best to cues with some significance (Kawasaki et al. 2001). Differential firing is also observed to neutral cues that predict reinforcement. Cells in both rat and primate orbitofrontal cortex fire selectively to such cues in discrimination tasks (Rosenkilde et al. 1981; Schoenbaum and Eichenbaum 1995a; Critchley and Rolls 1996a; Tremblay and Schultz 1999; Yonemori et al. 2000), and this se-

LEARNING \& MEMORY 8:134-147 @ 2001 by Cold Spring Harbor Laboratory Press ISSN1072-0502/01 \$5.00

$$
\begin{array}{llllllllllllllll} 
& E & A & R & N & I & N & G & \mathbf{Z} & M & E & M & O & R & Y \\
\text { www.learnmem.org } & & & &
\end{array}
$$


lective activity appears to reflect their reinforcement contingencies rather than their sensory features (Fig. 1), although some aspects of sensory features of the cues may be encoded as well (see Fig. 1, last panel).

Recent findings have expanded our understanding of this activity in the orbitofrontal cortex. These data indicate that although neurons in this prefrontal region are indeed preferentially involved in representing the significance of cues, they do not simply encode the associated reinforcer. Rather, these cells seem to selectively encode relevant incentive or motivational features of cues according to the context of ongoing behavior. Such performance-relevant representations in effect link affective information to ac-
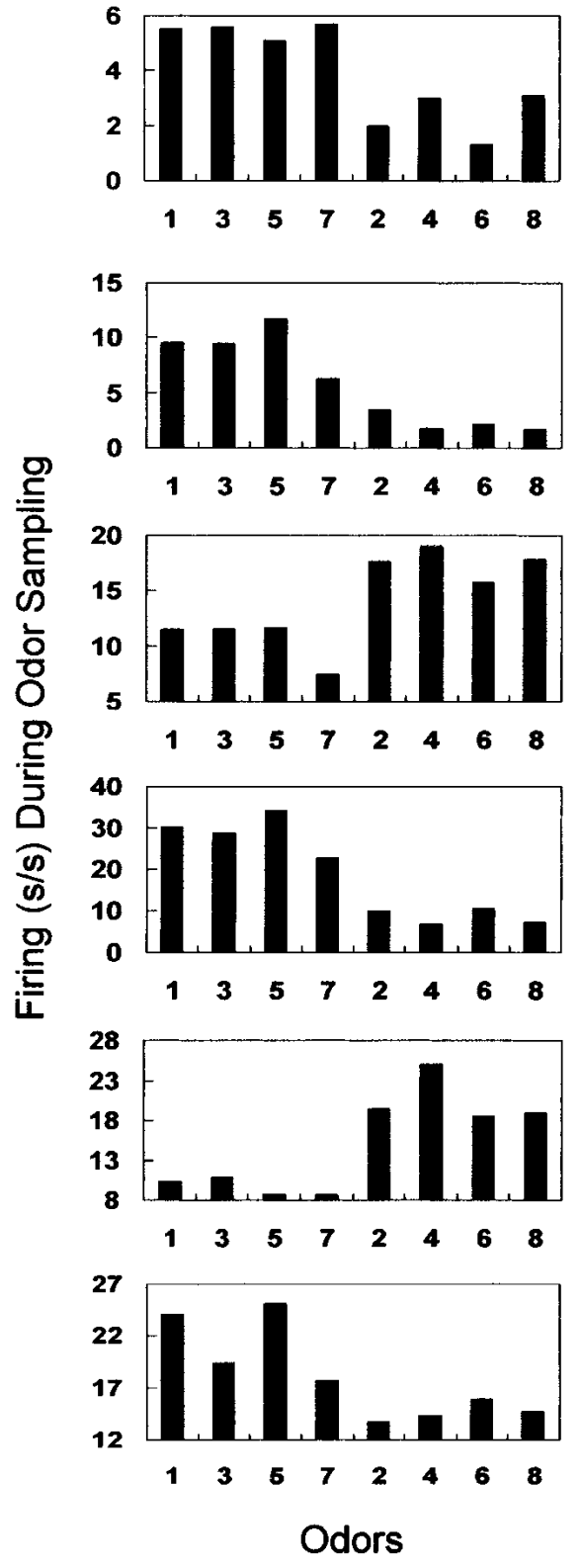

tion, and could represent rules that are used to guide goaloriented behavior. In addition, the manipulation of information to construct rule representations necessitates a working memory function. Consistent with such a function, representations of incentive value are also found during delays. Thus, neurophysiological data from both rats and primates indicate that prefrontal functions, including working memory and performance-relevant encoding, may be integrated in the orbitofrontal cortex as they are in the dorsolateral prefrontal cortex, thus providing some features of processing that are common across prefrontal subdivisions (Rosenkilde 1979).

\section{Neurons in the Orbitofrontal Cortex Encode the Acquired Incentive Value of Cues}

Although neurons in the orbitofrontal cortex do appear to represent associations between cues and reinforcers, a model that simply implicates the orbitofrontal cortex in representing such associations would be incomplete. After all, much behavior that depends on these associations is left unimpaired following a lesion to the orbitofrontal cortex. Conversely, some of the impairments caused by such lesions do not appear to result from deficits in associative representations. For example, patients with orbitofrontal lesions show impulsive behavior and poor decisionmaking. (Harlow 1868; Damasio 1994). This function of the orbitofrontal cortex has been formally implicated in performance of a gambling task, in which subjects must choose from decks of cards associated with rewards and penalties of different sizes (Bechara et al. 1997). Optimal performance requires that subjects learn the rewards and penalties associated with the different decks and then bias their choices based on this information to avoid large penalties. Patients with orbitofrontal damage are impaired on this task, while

Figure 1 Encoding of associated outcome rather than sensory features of cues in the orbitofrontal cortex revealed by neural activity during odor sampling. Neurons in rat orbitofrontal cortex were recorded during performance of an eight-odor go/no-go odor discrimination task. On each trial, one odor was presented. After odor sampling, a response could be made at a nearby fluid well for a water reward. Four odors were positive, indicating that a response would be rewarded, and four odors were negative, indicating that no reward would be given for responding. Rats were well-trained on the discrimination prior to recording, always responding to positive odors and rarely to negative odors. Activity is shown in spikes/second during odor sampling for six different neurons recorded in the orbitofrontal cortex. Each panel shows activity for a different neuron to each of the eight different odors presented in each session. Positive odors $1^{+}, 3^{+}, 5^{+}$, and $7^{+}$are on the left of each panel, and negative odors $2^{-}, 4^{-}, 6^{-}$, and $8^{-}$are on the right of each panel. Note that although some cells responded to all positive or all negative odors equally (panels 1-4), others responded best to a subset of the positive or negative odors (panels 5 and 6). (Reprinted, with permission, from Schoenbaum and Eichenbaum 1995b.)

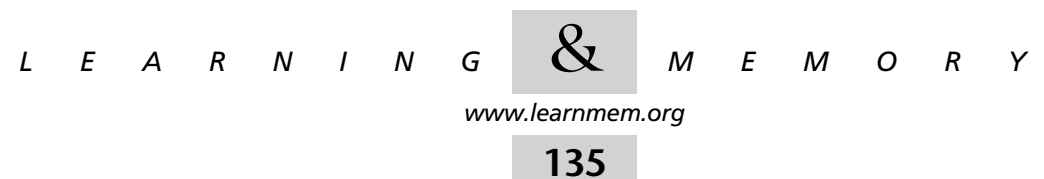


showing normal performance on other measures of prefrontal function (Bechara et al. 1998). In addition, regions within the orbitofrontal cortex exhibit marked hemodynamic changes correlated with the rewarding and punishing outcomes in versions of this task (Rogers et al. 1999; O'Doherty et al. 2001), and cells recorded from depth electrodes in human orbitofrontal cortex also appear to be activated in this task (Adolphs et al. 2000). Nevertheless, in many cases patients with orbitofrontal damage are able to verbalize the outcomes associated with the different decks of cards, demonstrating that they have acquired some information about the associations between the cards and their outcomes. Similarly, humans, nonhuman primates, and rats with orbitofrontal lesions can show intact performance on discriminations that require responding to neutral cues that predict reinforcement, while exhibiting marked impairment on reversals that presumably depend on similar information (Rolls et al. 1994; Meunier et al. 1997; Saddoris et al. 2001). Orbitofrontal lesions also affect performance in socalled devaluation paradigms. In these tasks, animals (rats or primates) with orbitofrontal damage respond normally to associations between cues and food reinforcers, but fail to modify their behavior to the cues when these representations are probed by reducing the incentive value of the food (Butter et al. 1963; Gallagher et al. 1999; Baxter et al. 2000). Similar to patients with orbitofrontal damage, these animals can acquire associative information regarding the cues but are not able to modify their responding to reflect that knowledge (Box 1).

These results indicate that processing of information in the orbitofrontal cortex is not simply related to encoding associations between stimuli and reinforcers. Rather, it may be that firing in the orbitofrontal cortex in response to cues represents, in part, information regarding the current incentive value of the associated reinforcer. This suggestion reflects the connections of the orbitofrontal cortex with the basolateral amygdala (Krettek and Price 1977; Price et al. 1987; Kita and Kitai 1990; McDonald 1991; McDonald et al. 1996), and the observation that, on identical tasks, lesions of the amygdala frequently result in impairments similar to those caused by orbitofrontal lesions (Jones and Mishkin 1972; Gaffan and Murray 1990; Hatfield et al. 1996; DeCoteau et al. 1997; Malkova et al. 1997; Bechara et al. 1999). Although changes in the incentive value of reinforcers alter firing in the orbitofrontal cortex to sensory properties of primary reinforcers in rats (D. Katz, pers. comm.) and primates (Critchley and Rolls 1996b), recording studies have only recently examined the relationship between differential firing in the orbitofrontal cortex to cues that predict reward (such as that illustrated in Fig. 1) and the value of the associated reinforcer (Rolls et al. 1996; Schoenbaum et al. 1999; Tremblay and Schultz 1999).

In these studies, one approach has been to assess neural firing to cues as an animal's preference for the associated reinforcer changes. Schultz and colleagues have done this by recording as monkeys are presented with discriminations involving differently-preferred reinforcer pairs. They found that cue-selective firing activity in the orbitofrontal cortex changed across blocks of trials in which different pairs of cues and reinforcers were presented (Tremblay and Schultz 1999), and that the changes in selectivity correlated with reinforcer preference, which was defined prior to recording in a choice version of the task. For example, a neuron that fired selectively to a cue paired with the preferred reinforcer in one block would decrease firing to this cue and switch selectivity if it became the nonpreferred cue in a subsequent trial block (Fig. 2). These data demonstrate that firing to discriminative cues in the orbitofrontal areas does not represent reinforcer identity, but instead correlates with the current incentive value of the reinforcer.

Another approach has been to reverse previously learned reinforcement contingencies in discrimination tasks. Rapid acquisition of reversals is dependent on the orbitofrontal cortex in both rats and primates (Rolls et al. 1994; Meunier et al. 1997; Saddoris et al. 2001), and cueselective firing in both rat and primate orbitofrontal cortex is severely affected when the cues associated with appetitive and aversive reinforcers are switched (Thorpe et al. 1983; Rolls et al. 1996; Schoenbaum et al. 1999). Indeed, the majority of cue-selective neurons in both species respond to reversal by becoming nonselective. In rats, activity in these cells no longer discriminates between the cues after reversal (Fig. 3A), not even when rats reach criterion performance on the reversed discrimination (Fig. 3B). These data are consistent with the proposal that this activity encodes incentive value and also demonstrate that encoding of incentive value is linked to specific cues. In other words, the firing represents the specific conjunction between a particular cue (or range of cues) and the current incentive value of an associated reinforcer. Similar conjunctive encoding is evident during reversal in primates (Thorpe et al. 1983). These conjunctions are disrupted by reversal of the reinforcement contingencies, causing the cells encoding them to become nonselective; a largely separate population of cells represents the new conjunctions after reversal (Schoenbaum et al. 1999). As we have noted, the orbitofrontal cortex appears to be crucial for using information regarding the acquired incentive value of cues to guide responses (Box 1).

\section{Neurons in the Orbitofrontal Cortex Encode Incentive Value When Relevant to Performance} Unlike dorsolateral prefrontal subdivisions, the orbitofrontal areas are implicated in emotion, social behavior, and decisionmaking (Damasio 1994; Bechara et al. 1997). These functions appear to be unique to the orbitofrontal regions (Bechara et al. 1998) and may depend on strong reciprocal connections with the amygdala (Krettek and Price 1977;

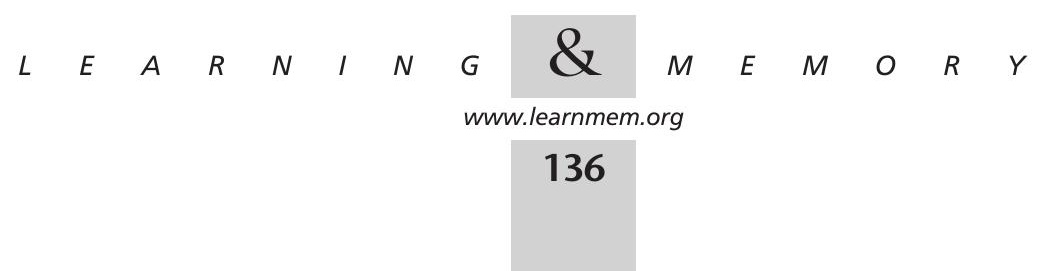




\section{Box 1. The Orbitofrontal Cortex and the Problem of Stimulus-Reinforcement Associations}

As Jones and Mishkin (1972) noted, the orbitofrontal cortex seems to be somehow involved in mediating behavior based upon stimulusreinforcement associations. However, associating stimuli with important events in some form is unlikely to be localized to a single brain structure. Rather, the ability to associate stimuli is probably a more general property of the brain, and responses are likely to be supported by several types of associative processes. For this reason, it is difficult to assess the contribution of a single brain area to this process, which may be why in subsequent testing many reports have failed to find discrimination deficits from lesions of orbitofrontal cortex (Rolls et al. 1994; Meunier et al. 1997; Saddoris et al. 2001). One paradigm that has been more successful is based on changing the incentive value of the associated reinforcer (Gallagher and Holland 1999).

In one version of this task, a hungry rat is trained to associate a conditioned stimulus (CS), such as a light, with a food unconditioned stimulus (US) (Fig. B1A). Training results in the emergence of a conditioned response to the CS based on several types of associations, one of which is between the CS and the incentive value of the US (Hatfield et al. 1996). Subsequently, the incentive value of the food item is "devalued" outside of the training situation by pairing it with illness (Fig. B1B). When the rat is then reexposed to the CS in the training environment (in the absence of food presentation), conditioned responses are altered to reflect the new value of the food (Fig. B1C). An important point to note is that several associative processes (e.g., associations between the CS and the unconditioned response elicited by the food or associations between the CS and the incentive value of the food) could potentially be used to guide conditioned responding. However, in order to modify its responding to the CS following devaluation, the rat must be able to access an association between the CS and the modified value of the food. A rat that is unable to access or use such associations to guide behavior will not show the normal change in responding observed in intact rats. This is precisely the pattern of deficit that we have found in rats with lesions of orbitofrontal cortex (Fig. B1C). Although these rats exhibit normal conditioned responses and respond normally to the devalued food item (i.e., they avoid eating it), they fail to exhibit the normal decrease in responding to the conditioned stimulus following food devaluation (Gallagher et al. 1999).

Similar results have been obtained in primates using an operant procedure in which a food item is devalued by selective overfeeding. Following this manipulation, monkeys normally change their responses to visual cues associated with the devalued food item. Monkeys with bilateral lesions of the orbitofrontal cortex or crossed lesions designed to disconnect the orbitofrontal cortex from the amygdala fail to show this change in response (Butter et al. 1963; Baxter et al. 2000; Izquierdo and Murray 2000). These data indicate that in both species, the orbitofrontal cortex is involved in representing or using the current motivational value of cues to guide behavior. Bilateral lesions of the amygdala (particularly the basolateral complex) cause similar deficits (Hatfield et al. 1996; Malkova et al. 1997). Despite the similarity in behavioral results, recent neurophysiological data indicate that functions of the two structures may be distinct in that the amygdala is preferentially involved in representing incentive value, whereas the orbitofrontal cortex uses such information to guide choice performance (Schoenbaum et al. 1999, 2000).

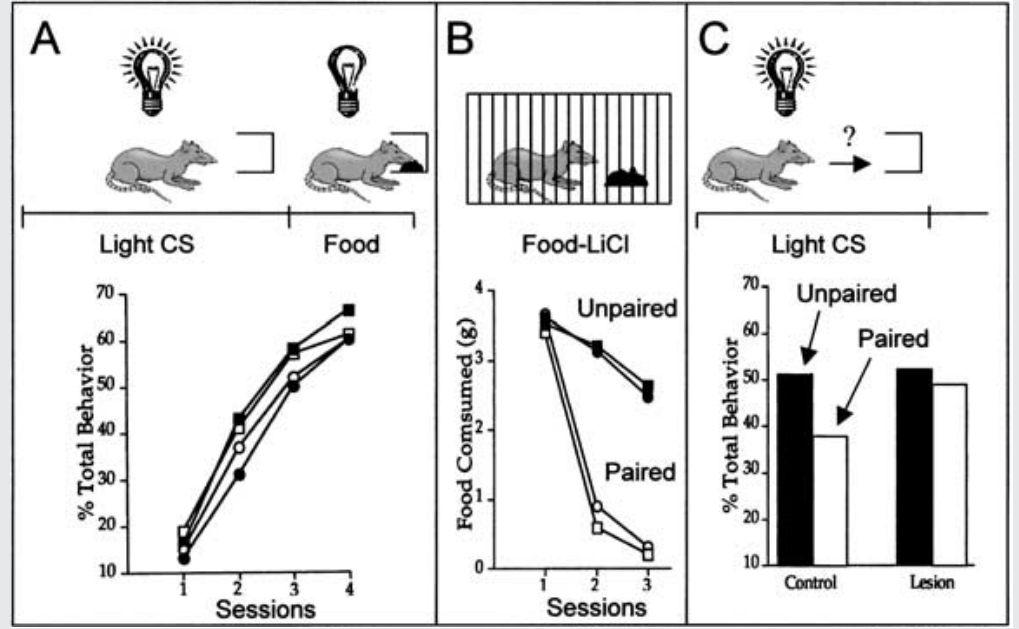

Figure B1 The effect of orbitofrontal lesions on conditioned responding after reinforcer devaluation. $(A)$ Food-deprived rats received conditioning sessions in which a light stimulus is paired with presentation of a sucrose food pellet. Both control rats (squares) and rats with bilateral neurotoxic lesions of the orbitofrontal cortex (circles) developed a conditioned response to the light (entry into the food cup). ( $B$ ) The rats then received presentations of the sucrose food pellets in their home cage followed by illness induced by lithium chloride injections. Fifty percent of the rats received paired presentations of food and illness (filled symbols), and the other half received unpaired presentations (open symbols). Both the control and the lesioned rats developed a taste aversion to the food item. $(C)$ The rats were then placed back into the training environment and their conditioned responses to the light were assessed in the absence of food presentation. Intact rats that received paired presentations of food and illness exhibited fewer conditioned responses to the light when compared to rats that received unpaired presentations. Rats with lesions of the orbitofrontal cortex continued to respond to the light after devaluation, even though they did not consume the food either in the home cage or in the training environment. In other words, the devalued representation of the food pellets failed to influence conditioned responding to the light. (Gallagher et al. 1999, reproduced by permission of the publisher.)

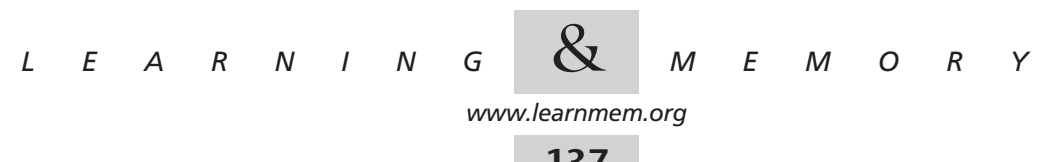


Price et al. 1987; Kita and Kitai 1990; McDonald 1991; McDonald et al. 1996). Studies linking neural activity in the orbitofrontal cortex to the acquired incentive value of cues confirm the unique role of this area of the prefrontal cortex in processing affective or motivational information attached to cues. However, if the orbitofrontal cortex is important for translating incentive value into goal-oriented behavior, one key question is the relevance of encoding that has been observed in this region to such behavior. Several recent experiments indicate that, in fact, encoding of incentive value in the orbitofrontal cortex is strongly correlated with behavior and task performance.

Initial recordings in rat orbitofrontal cortex showed that task variables in an eight-odor discrimination task were represented by the firing of the population according to the requirements of the task (Schoenbaum and Eichenbaum 1995a,b). As discussed earlier, neurons in the orbitofrontal cortex of well-trained rats fired selectively to the odor cues in this task (Fig. 1). Ensemble analyses (Fig. 4A) confirmed that activity in these neurons represented odor identity and valence (Fig. 4B), features that were useful in the performance of the task. In addition to these neural correlates, however, activity during odor sampling also reflected aspects of the odor sequence, both consequential and irrelevant. For example, some neurons fired differently to odors that predicted the odor of the following trial. This prospective encoding was reflected in the rat's behavioral performance; rats initiated trials more rapidly or more slowly when such odors indicated that a positive or negative odor could be predicted on the upcoming trial. Other neurons fired differently to a given odor depending on the valence of the previous trial. Such retrospective information had no bearing on the rat's performance in this task. Subsequent ensemble analyses of encoding of these features by these same neural populations revealed that the cells preferentially encoded the information that was behaviorally relevant. Irrelevant features of the odor sequence, such as the valence or identity of the odor on the previous trial, were poorly represented (Fig. 4C), but the predictive relationships between specific odors were well represented (Fig. 4D). Consistent with the preferential encoding of behaviorally relevant information, this information was well encoded by the ensembles prospectively (Fig. 4D, left panel) but not retrospectively (Fig. 4D, right panel). In other words, information conveyed by the odors was represented in the orbitofrontal cortex according to its usefulness in the task.

If neural activity reflects the use of incentive value information in guiding behavior, then changes in discriminative behavior should be reflected in the development of
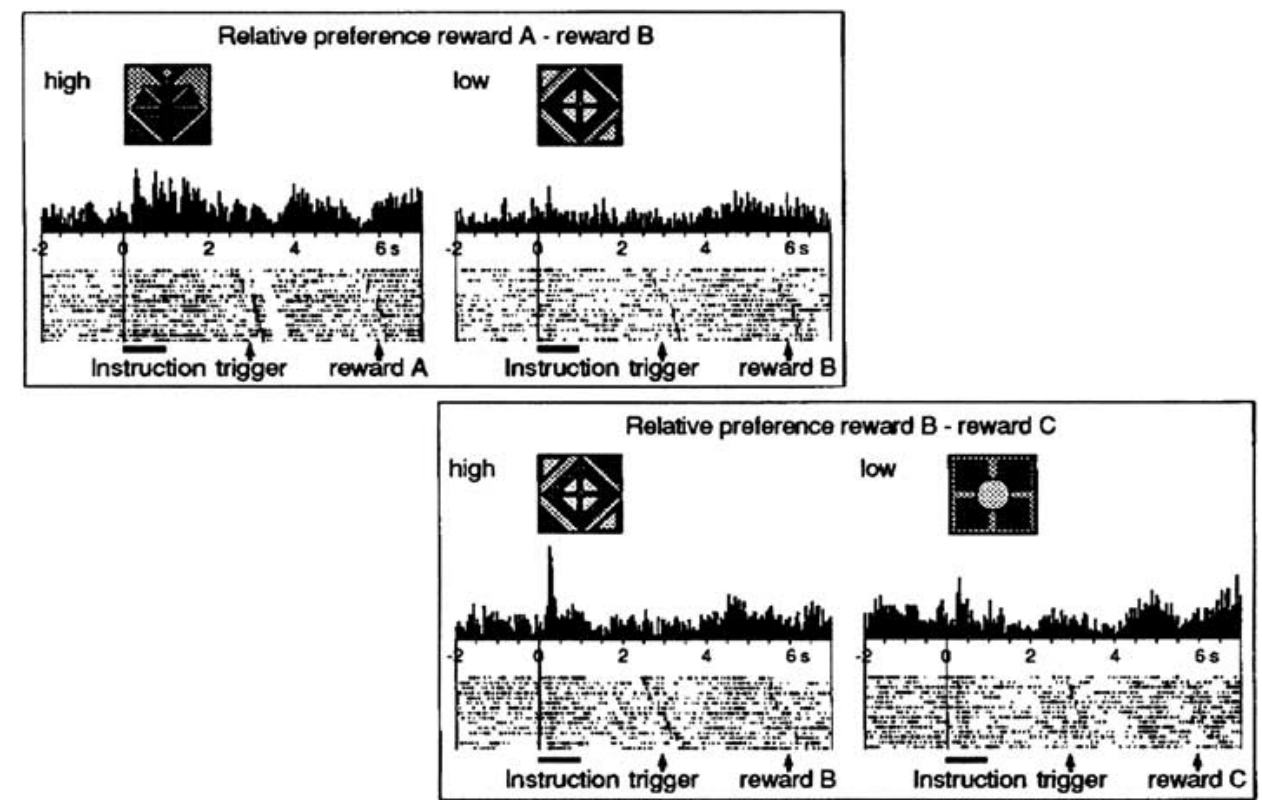

Figure 2 Representation of incentive value in primate orbitofrontal cortex revealed by correlations between neural activity and relative preference among available reinforcers. The neuron was recorded as the monkey performed a visual discrimination task. Pictures (shown) were associated with delivery of different rewards, and blocks of trials differed in which two pictures were shown. This neuron exhibited increased activity during presentation of the pictures and changed firing across blocks in which different rewards were available. In the first block of trials represented in the upper rasters, the neuron fired more during presentation of the cue associated with reward $\mathrm{A}$ (raisin) than during presentation of the cue associated with reward B (apple). This selectivity mirrored the preference of the monkey for raisin over apple determined in separate choice testing. In a subsequent block of trials represented in the lower rasters, the cue associated with reward B (apple) was presented with a cue associated with reward C (cereal). The neuron altered its firing selectivity to reflect the new relative preference of the monkey for apple over cereal. (Schultz et al. 2000, reproduced by permission of Oxford University Press.)

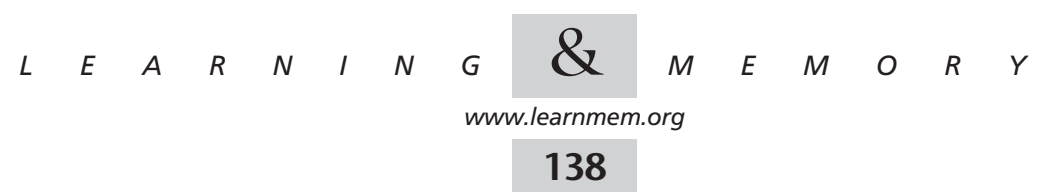


encoding in the orbitofrontal cortex. To test this hypothesis, we recorded in rat orbitofrontal cortex during learning of new odor discrimination problems (Schoenbaum et al. 1999). Discriminations involved either two or four novel odors paired with sucrose or quinine. We examined activity in a population of neurons similar to those from earlier work (Fig. 1). Activity in these neurons was examined across several phases in each session related to learning and choice performance (Fig. 3C). We found that the vast majority (91\%) of orbitofrontal cortex neurons with selective firing during stable performance on the task only developed

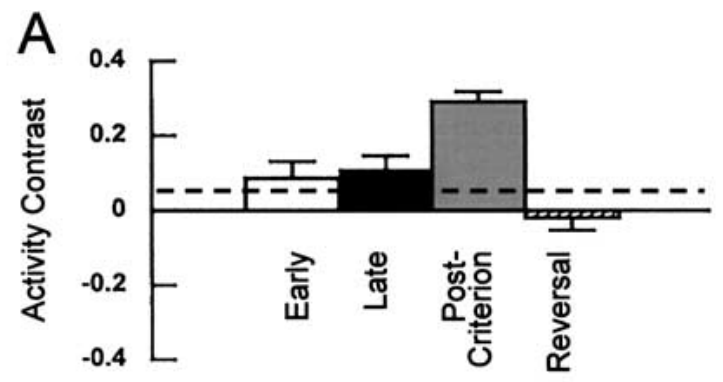

B

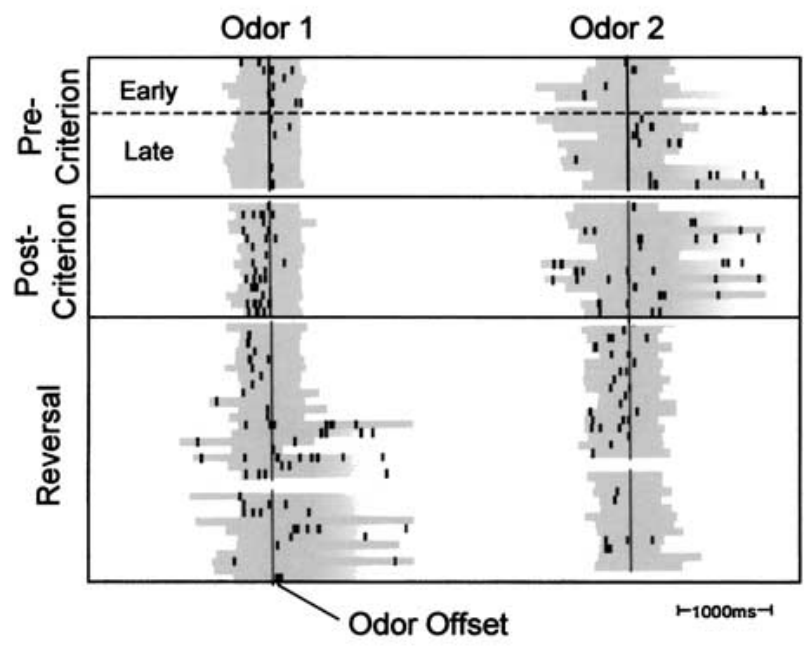

C

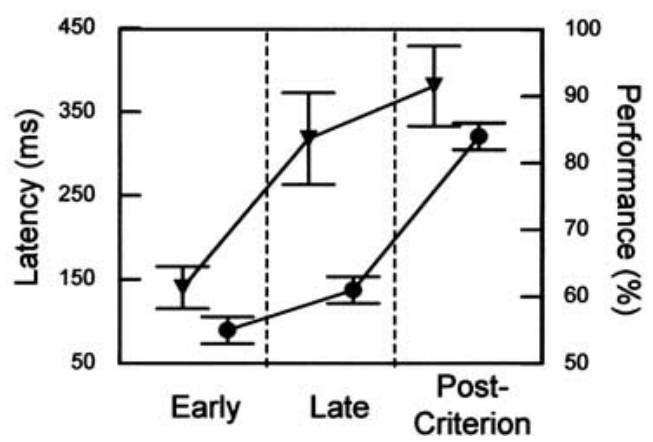

that selectivity during highly accurate performance (postcriterion, Fig. 3A). In other words, these cells did not fire selectively at the start of the session when the odors were novel (early phase, Fig. 3A), nor did they fire selectively during a later phase of training (late phase, Fig. 3A) characterized by chance performance on the discrimination but development of long-latency responses to negative cues (Fig. 3C). Similar changes in response latency have been shown to be related to the incentive value of neutral cues paired with reinforcers (Holland and Straub 1979). Yet in our paradigm changes in response latency are not reflected in population firing activity in the late phase when choice performance is unchanged. As the example illustrates (Fig. 3B), selectivity only emerged during postcriterion perfor-

Figure 3 Representation of the acquired incentive value of cues in rat orbitofrontal cortex revealed by neural activity during discrimination and reversal training. Neurons in rat orbitofrontal cortex were recorded during acquisition and reversal of new two-odor discrimination problems in a go/no-go paradigm. On each trial, an odor was presented. Responses after sampling of the positive odor resulted in delivery of a sucrose solution; responses after sampling of the negative odor resulted in delivery of a quinine solution. Rats were presented with a novel odor pair in each session. Neural activity was recorded as the rats learned to withhold responses to the negative cue to avoid the quinine solution, during accurate performance (post-criterion), and during reversal training in which the reinforcers paired with the odor cues were switched. (A) Contrast in population activity during odor sampling calculated for the 96 of 328 orbitofrontal neurons that exhibited selective firing to odor cues during accurate performance. Activity contrast was calculated as the difference in firing to positive and to negative odors, referenced to the polarity of this difference during post-criterion trials, and normalized by the sum of those rates in each training phase. The dotted line represents a baseline value from shuffled trials. Selectivity in this population of neurons differed significantly from baseline only during the post-criterion phase, indicating that differential activity in these cells (illustrated in $B$ ) only developed in the post-criterion phase of pre-reversal training and disappeared after reversal. (B) An example of a neuron with differential firing during odor sampling that developed during accurate performance of the discrimination and disappeared following reversal. Neural activity is shown for representative trials in raster format. Trials are shown sequentially for each odor. Activity on each trial begins with odor onset, is synchronized to odor offset, and ends with a response or after $1500 \mathrm{~ms}$ for no-go trials (faded). This cell develops selective firing to odor 1 in the post-criterion trial block, during accurate performance on the discrimination. This selective response diminishes rapidly after reversal and, in fact, disappears. Note that selectivity does not reemerge when the criterion is met on the reversal (rasters following break in reversal section) and thus does not simply reflect the discriminative motor response or reinforcer identity. $(C)$ Changes in behavioral measures during acquisition in the sessions represented in $A$. Response latency and choice performance were measured, and acquisition was divided into three phases that differed in these performance measures: an early phase, a late phase, and a post-criterion phase. Note the divergence between the difference in response latency on positive and negative trials (triangles) and performance (circles) in the late phase of pre-criterion training, indicating learning regarding the associations between the odors and outcomes without a shift in choice behavior. (Reprinted, with permission, from Schoenbaum et al. 1999, 2000.)

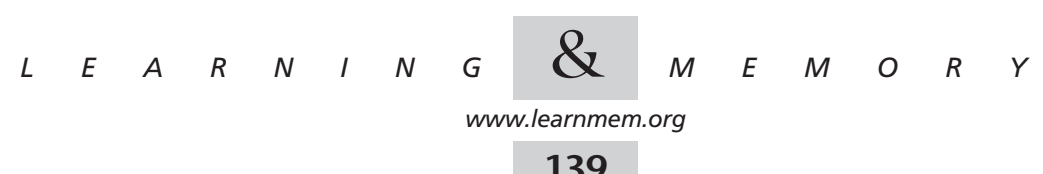



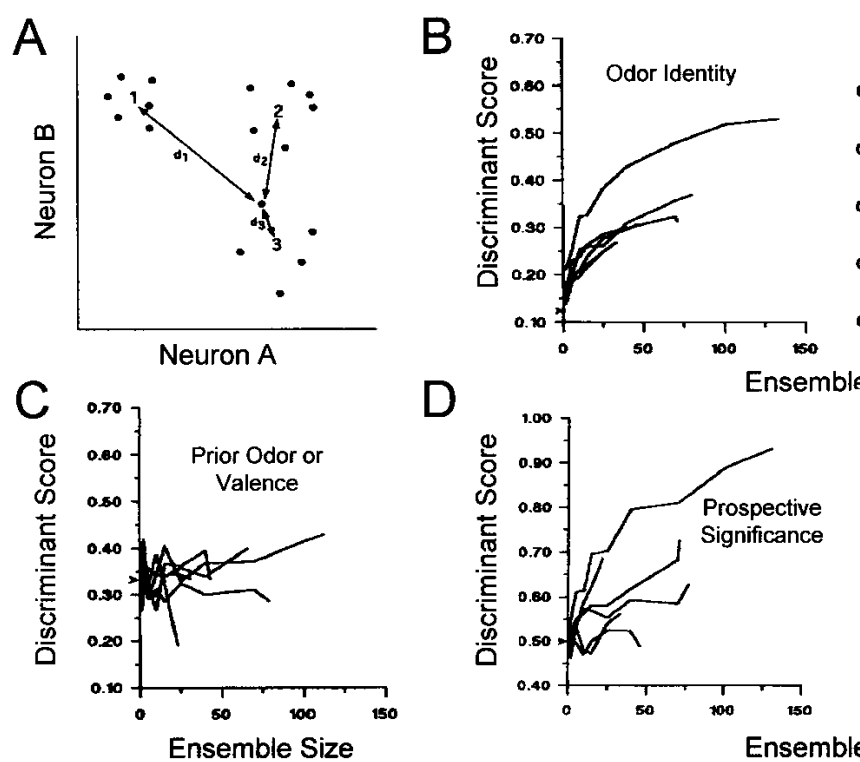

Figure 4 Encoding in orbitofrontal cortex during odor sampling in an eight-odor go/no-go discrimination task. A modified linear discriminant analysis (Gochin et al. 1994) was performed to determine how well activity in populations of odor-responsive orbitofrontal neurons in each rat could identify attributes of the odor cue on each trial and of the sequence of odor cues on preceding or subsequent trials. (A) Illustration of ensemble analysis. This analysis creates a space using the activity of each neuron as one of $n$ dimensions. The population response on each trial is plotted in this space, and then the average population vector $(1,2,3$, or positive versus negative, etc.) for each item in a discrimination (odor, valence, predictedness, etc.) is determined. The population vector for the response on each trial is then classified as belonging to the nearest cluster in this space by calculating the distance to each average vector $\left(d_{1}, d_{2}, d_{3}\right)$. The discriminant score is calculated by comparing the classification of each trial to the actual identity. ( $B$ ) Discriminant score for ensembles of orbitofrontal neurons during discrimination performance. Populations were composed of odor-responsive neurons. Individual neurons responded to both odor identity and valence, and ensembles performed well at correctly classifying odors according to these attributes. (C) Other neurons fired differentially depending on the odor presented on the preceding trial or on whether the prior trial had been rewarded or not. Such incidental information was irrelevant to task performance, and ensembles performed at chance at identifying these attributes. $(D)$ Neurons also fired differently according to fixed pairs of odors that were always presented in sequence. This information was reflected in the latency to initiate the next (predicted) trial, and the ensembles performed well at discriminating between predictive and nonpredictive odors (prospective encoding, left panel in $D$ ). However, ensembles performed poorly at classifying whether an odor had been predicted (retrospective encoding, right panel in $D$ ). (Reprinted, with permission, from Gochin et al. 1994; Schoenbaum and Eichenbaum 1995b.)

mance when choice performance changed to reflect the information provided by the odor cues. These data indicate that the firing changes in the orbitofrontal cortex may be separated from other behaviors dependent on incentive value. Furthermore, as mentioned earlier, selective firing in the majority of these neurons was disrupted by reversal of the reinforcement contingencies of the odor cues (Fig. $3 \mathrm{~A}, \mathrm{~B})$. This population was, in effect, replaced by a separate group of neurons ( $\sim 25 \%$ of the remaining cells) that became selective after reversal. Thus, the selective activity did not simply reflect the discriminative response or motor set but, rather, reflected the linkage of the acquired incentive value of the specific odor cues with the use of that information to guide choice performance.
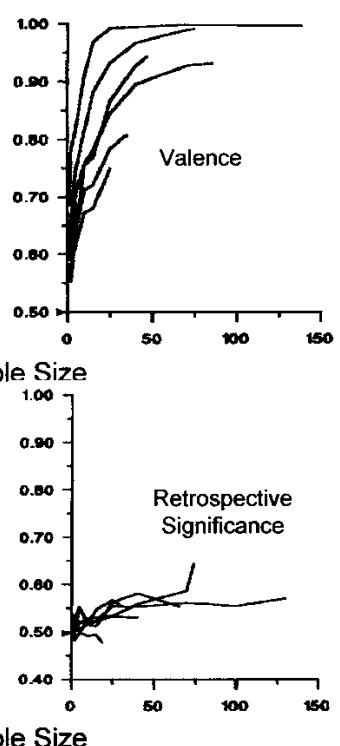

If firing to odor cues in orbitofrontal cortex represents information that is relevant to task performance, then one would predict (Schoenbaum and Eichenbaum 1995b; Schoenbaum 1998) that correlations to odors would be different if the way in which odors were used to guide performance were different. This prediction is borne out in a recent study by Ramus and Eichenbaum (2000). In this study, rats sampled an odor on each trial and were rewarded for responding if the odor did not match the odor sampled on the immediately preceding trial (continuous delayed-nonmatch to sample, or cDNM, task). Although odor identity was important in determining whether a match or nonmatch was made, the identity of the odor was not, by itself, correlated with outcome. Thus, the rule guiding the odor-based performance differed from that in the discrimination task. Notably, orbitofrontal cortex lesions can affect the performance of rats in both tasks (Eichenbaum et al. 1983; Otto and Eichenbaum 1992).

In this context, in which a given odor could be rewarded on one trial but not on the next, selective firing to any of the eight different odors was observed in $<15 \%$ of the orbitofrontal cortex neurons (43 neurons) compared to $77 \%$ of neurons in the eightodor discrimination task. In addition, many odor-selective cells (30 of 43 neurons) actually fired differentially to a particular odor based on whether it was a match or a nonmatch presentation, rather than on the basis of the odor identity (Fig. 5A). In fact, nearly $64 \%$ of the orbitofrontal cortex neurons recorded in this task (175 of 276 cells) fired differently to the same group of odors when match and nonmatch presentations were compared, and there was a strong correlation between performance in different sessions and the proportion of cells with match/nonmatch correlates and no correlation with representation of odor (Fig. 5B). In contrast, such retrospective information about odor sequence was represented at the level of chance during discrimination performance (Fig. 4C; right panel, Fig. 4D), and odor identity was well represented (Fig. 4B).

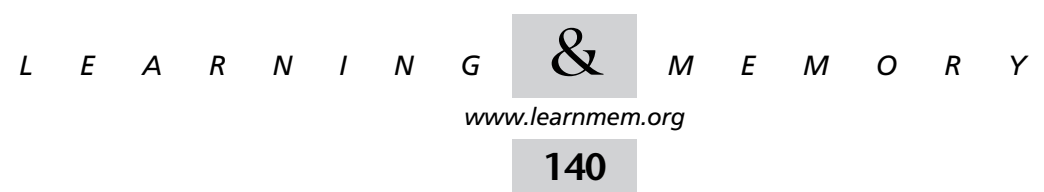




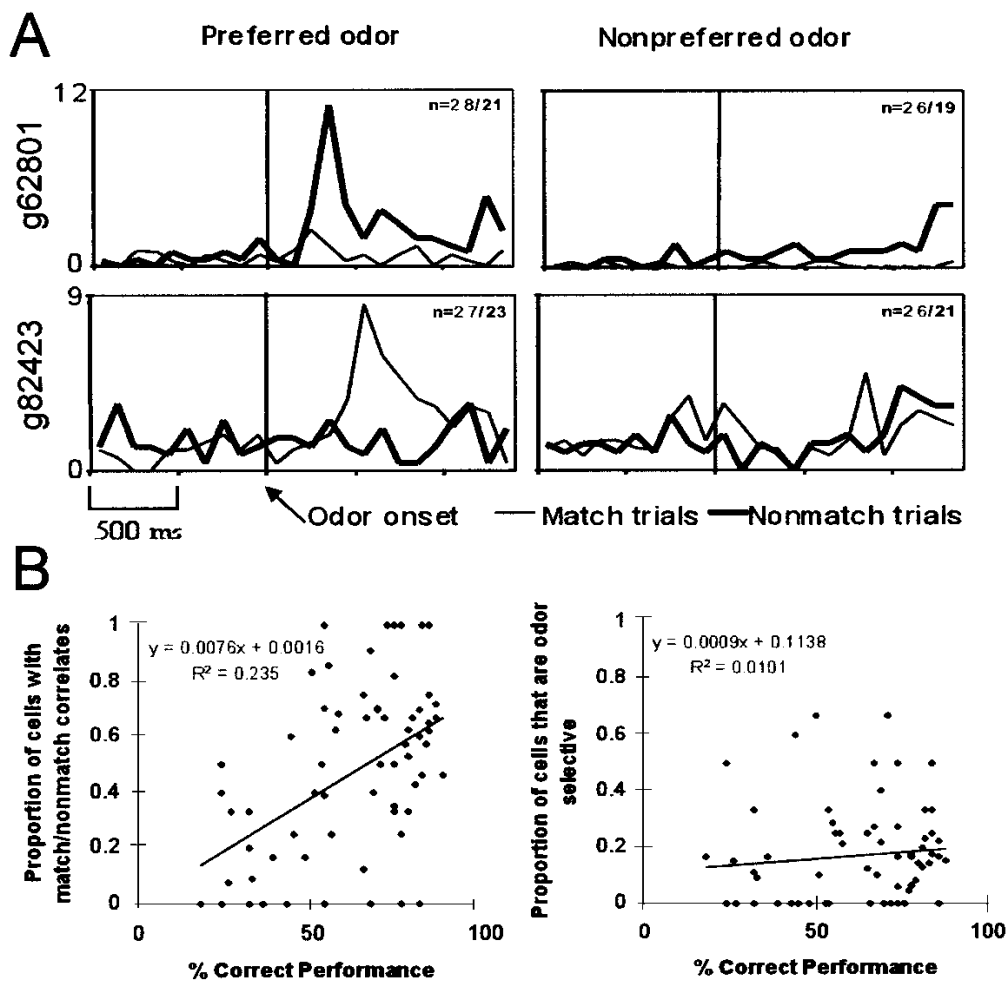

Figure 5 Encoding in orbitofrontal cortex during odor sampling in a CDNM (çontinuous delayed nonmatching to sample) task. (A) Average activity is shown in two neurons recorded in orbitofrontal cortex (g62801 and g82423) to the most and least preferred of the eight odor cues presented in each session. In addition, activity to each odor is plotted separately according to whether the odor matched (-) or did not match (+) the odor cue presented on the preceding trial. Note that although these two neurons appear to be odor-selective, the selectivity is entirely dependent on the match, nonmatch comparison. Neuron g62801 exhibits the same low level of firing to a match presentation of the preferred odor as it does to presentations of the nonpreferred odor. Similarly, neuron g82423 fires no more to a nonmatch presentation of the preferred odor than to presentations of the nonpreferred odor. (B) Encoding versus session performance during odor sampling in the cDNM task. The importance of the match, nonmatch comparison (and the relative unimportance of the odor identity) is illustrated by plotting the correlation between performance in a session and the proportion of neurons with match/nonmatch or odor-identity correlates. Only the proportion of the match/nonmatch correlates increases with performance. No other category of correlate was related to performance in this task, in marked contrast to the importance of odor identity and other features in an odor discrimination task (see Fig. 4). (Reprinted, with permission, from Ramus and Eichenbaum 2000.)

In summary, when odor-guided performance was based on matching comparisons, orbitofrontal cells responded differently according to whether an odor was a match or nonmatch presentation. Moreover, encoding developed with accurate choice performance, as was observed in the orbitofrontal cortex during go/no-go odor discrimination training (Schoenbaum et al. 1999). Although this first report of activity in orbitofrontal cortex in the cDNM task did not separate learning from changes in choice performance, we predict that more subtle changes in behavior (such as response latency), based on the associations between match or nonmatch odor presentations and outcomes, were present in earlier sessions in which choice performance was poor and match/nonmatch correlates were rare. Nevertheless, the findings are consistent with our earlier report indicating that information regarding the acquired incentive value of cues or cue configurations is encoded when relevant to task performance.

Considered together, these two studies in rat orbitofrontal cortex are similar to recent reports of encoding of visual cues in primate dorsolateral regions (Rainer et al. 1998; Asaad et al. 2000). Although earlier reports of activity in these regions stressed segregation of encoding properties according to anatomical location (Wilson et al. 1993), these more recent studies found that encoding properties were also related to task demands. Cells responsive to object identity were prevalent when the discrimination performance of the monkey was based on associations between specific objects and outcomes, whereas cells responsive to object location or place were prevalent when the monkey's discrimination performance was based on associations between these cue features and outcomes. These findings and those in the orbitofrontal cortex indicate that across species and across subdivisions, processing in the prefrontal cortex may be characterized by encoding of specific attributes of cues according to the use of these attributes to guide task performance. In the dorsolateral regions, the cue attributes are best defined by physical features such as object identity, color, or location. In the orbitofrontal regions, cue attributes are related to acquired motivational or incentive value.

Task- or performance-relevant processing bears a striking similarity to the concept that the prefrontal cortex represents rules that can be applied to guide behavior. Proponents of rule representation (Dias et al. 1996; Wise et al. 1996; Wise and Murray 2000) hold that the prefrontal cortex is specialized to identify or acquire rules that allow appropriate behavior in different circumstances or contexts. Prefrontal mechanisms are thought to be particularly important when conflicting priorities or contexts must be resolved as in reversal tasks or social interactions, but one would expect that they would be operative in all situations (Cohen et al. 1998). If this were true, one might expect firing activity in prefrontal subdivisions to reflect the linkage of specific information, such as incentive value or features of visual cues, to appropriate behavior. This, of

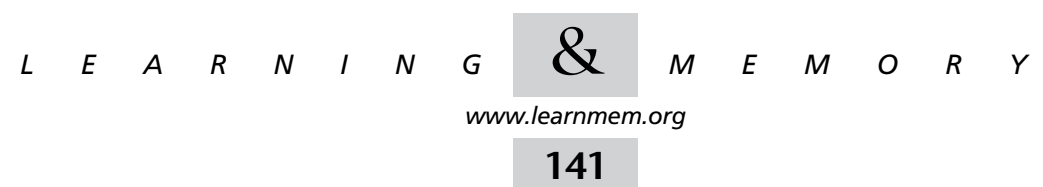


course, is precisely what we and others have observed in the orbitofrontal cortex.

One implication of the idea that prefrontal areas, including the orbitofrontal cortex, display rule-based encoding is that the constituent elements that make up the rule are accessed or represented independently (Schoenbaum 1998). For example, if an animal chooses not to respond to a cue to obtain a reward after selective satiation of that reward, then the rule guiding behavior has changed but the association between the cue and the reward has not. The orbitofrontal cortex must access that information from elsewhere in the brain when evaluating the context (satiation) and making the decision not to respond. Accessing and manipulating that information to construct the neural representation appropriate for a given context requires a working memory function. Such a working memory function is, of course, readily apparent for visual attributes of cues in studies of primate dorsolateral cortex (Wilson et al. 1993). Next we present data that argue for a similar function in the orbitofrontal regions of the prefrontal cortex.

\section{Neurons in the Orbitofrontal Cortex Encode Incentive Value During Delays}

To some extent, the wide acceptance of the idea that the dorsolateral prefrontal cortex is involved in working memory comes from the demonstration that cells recorded in this region in visually cued delayed-response tasks exhibit selective activity during the delay phase (for examples, see Fuster et al. 1982; Funahashi et al. 1989; Wilson et al. 1993; Rainer et al. 1998). Animals with dorsolateral prefrontal cortical lesions are impaired on these tasks, and neurons in this region fire selectively to features of the visual cues that the animal must remember across the delay, such as the color, identity, or location of the particular item that was shown. Careful examination has shown that a large proportion of these neurons do, indeed, represent these attributes of the cue rather than encoding the motor response. Delay-selective neural activity has also been reported in the orbitofrontal cortex, but unlike neural activity in dorsolateral regions, activity in the orbitofrontal areas represents the incentive value or significance of the cues during delays.

Orbitofrontal neurons are active in both rats and primates during short delays interposed between responses and reinforcer delivery (Schoenbaum et al. 1998; Tremblay and Schultz 1999, 2000a) or during delays between cue presentation and responses to instructions preceding reinforcer delivery (Hikosaka and Watanabe 2000). For example, in rats learning new odor-discrimination problems (Schoenbaum et al. 1998), orbitofrontal neurons exhibit activity during a delay prior to reinforcement (Fig. 6). This activity appears to encode expectancy of the impending outcome, independent of the response made to gain access to the outcome. Activity differs when the same response is made but different reinforcers are anticipated. Similar find-

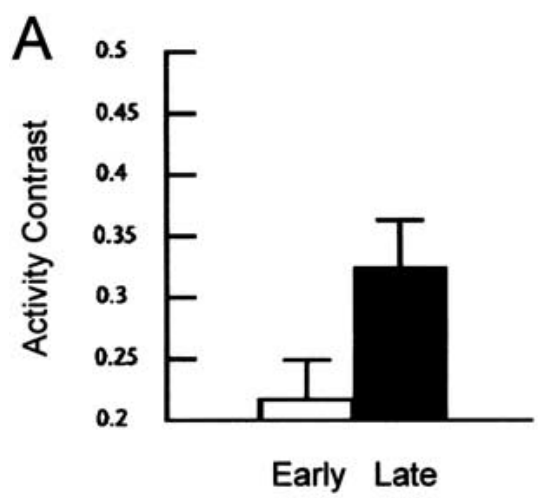

8

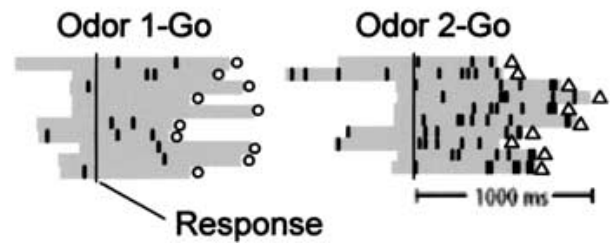

Figure 6 Representation of incentive value in the orbitofrontal cortex during a delay after cue sampling and response but before reinforcer delivery. Neurons were recorded in the same experiment described in Figure 3. (A) Contrast in population activity during the delay after responding at the fluid well but before reinforcer delivery, calculated for the 74 of 328 orbitofrontal neurons that exhibited selective firing during this period (illustrated in $B$ ). Selective firing during the delay in this population of neurons developed rapidly during training and increased significantly with learning between the early and late phases of pre-criterion training. Activity contrast was calculated by comparing firing during the delay on positive and negative trials as described for Figure 3. (B) Example of a neuron that fired differentially during a brief delay after a response but before reinforcer delivery. The cell fired more during the delay on trials involving odor 2 (associated with quinine) than on trials involving odor 1 (associated with sucrose). This selective activity appears to anticipate delivery of the quinine solution (triangles) but not sucrose (circles). Neural activity is shown for representative trials in raster format. Trials are shown sequentially for each odor. Activity on each trial begins with odor offset, is synchronized to the response at the fluid well, and ends with delivery of sucrose (circles) or quinine (triangles). Only go responses made during pre-criterion training are shown. (Reprinted, with permission, from Schoenbaum et al. 1998.)

ings have been made in primate orbitofrontal cortex (Tremblay and Schultz 1999). In that report, differential firing was not observed during delays when a response was made but no reward was expected (Tremblay and Schultz 2000a). Similar to activity during presentation of predictive cues, delay-related activity in the orbitofrontal cortex emerges with learning (Schoenbaum et al. 1998, 1999; Tremblay and Schultz 2000b) and is linked to the incentive value of the impending reward rather than to the specific identity of the reward (Tremblay and Schultz 1999; Hikosaka and Watanabe 2000). Similar encoding during delay periods or in anticipation of events has subsequently been reported in other

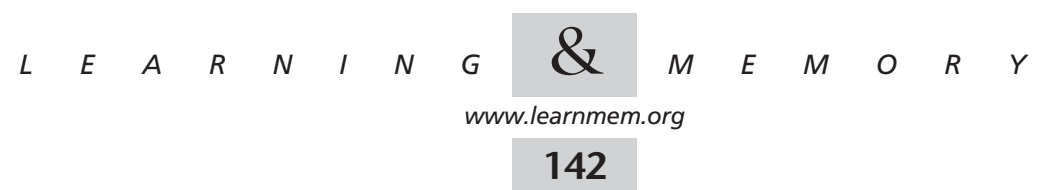


tasks in which predictions can be made regarding impending events or outcomes (Lipton et al. 1999). Thus, the orbitofrontal cortex may prospectively encode a variety of meaningful events in tasks.

The encoding of anticipated events by selective subsets of orbitofrontal neurons is particularly intriguing when compared to the orbitofrontal activation reported in imaging studies when expectations are not met (Nobre et al. 1999) or when guesses regarding likely outcomes must be made (Elliott et al. 2000). Although the hemodynamic changes that produce these signals likely reflect activity in many neurons (Scannell and Young 1999), the anticipatory encoding we and others report in specific subsets of cells may form the basis for the more massive activation seen with imaging techniques when outcomes deviate from expectations. Notably, the selective firing during delays is observed in rat orbitofrontal cortex early in learning and before changes in response selection are evident, suggesting that expectations are formed on the basis of even incomplete information. The resultant signal demonstrated in these studies where outcomes do not match expectations could represent an attentional function as inactive cells are engaged to encode new information. We observe just such a phenomenon following during reversal training (Schoenbaum et al. 1999).

Finally, although anticipatory encoding of reinforcers during delays is also observed in other prefrontal regions, the firing of delay cells in the orbitofrontal areas is related to the incentive value of the outcome rather than the specific identity of the reinforcer. Schultz and colleagues demonstrated this property in their recent work described above (Tremblay and Schultz 1999), in which monkeys were presented with a visual discrimination task involving several different visual cues paired with distinct and differentlypreferred reinforcers. Selective firing during a delay interposed between the response and reward delivery changed based on the monkey's relative preference between the reinforcers available in a given block of trials. In other words, a neuron would fire differently during a delay in anticipation of a reinforcer depending on whether it was the preferred or nonpreferred of the two in the trial block. Similar findings have since been reported during delays that precede responding as well (Hikosaka and Watanabe 2000). Those authors, in fact, compared delay-selective activity in the orbitofrontal and lateral prefrontal areas and found that selective firing during the delay was far more likely to represent reinforcer identity in the lateral prefrontal cortex, whereas similar activity in orbitofrontal regions typically did not discriminate between reinforcers except when food preferences changed during testing (Fig. 7). The dependence of these correlates on the incentive value of the reward therefore appears to distinguish anticipatory delay activity in orbitofrontal cortex from similar activity elsewhere in prefrontal cortex (Watanabe 1996; Hikosaka and Watan-

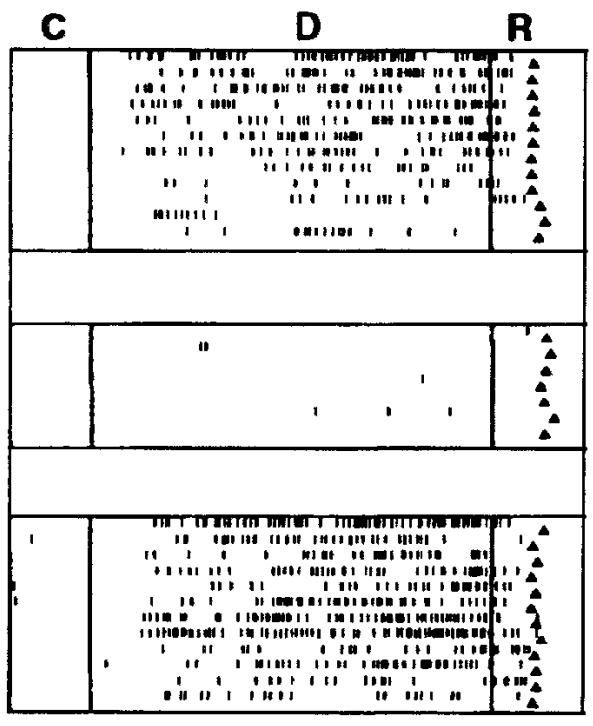

Figure 7 Representation of incentive value in the orbitofrontal cortex during a delay after cue sampling but before response to an instructional cue to receive reward. This cell was recorded in a monkey performing a visual discrimination task. Activity is shown in raster format. Each trial is divided into cue presentation (C), a delay phase (D), and reward (R). Reward is indicated by a triangle. The cell fired during the delay preceding reward in anticipation of raisin (top panel). Similar delay activity was evident on other trials (not shown) in anticipation of potato or cabbage. Later in the session, activity during the delay disappeared when the monkey stopped eating the raisin when it was presented (middle panel). Delay activity was observed on non-raisin trials (not shown). Firing during the delay resumed on raisin trials later in the session, when the monkey again began eating the raisin (bottom panel). (Reprinted, with permission, from Hikosaka and Watanabe 2000, Oxford University Press.)

abe 2000). Viewed from this perspective, it is worth noting that several studies have found delay-dependent effects of orbitofrontal cortex lesions, a hallmark of working memory in primates (Box 2).

\section{Conclusions}

Here we have reviewed behavioral and electrophysiological evidence from studies in rat and primate orbitofrontal cortex to argue that orbitofrontal function can be integrated into the framework of prefrontal theory. This evidence indicates that several features of encoding (which appear to be conserved across species) in primate dorsolateral prefrontal cortex may be generalized to explain encoding in the orbitofrontal region. The first of these is an integrative function operating across multiple phases of a task that results in the preferential representation of afferent information according to the information's relevance to task performance. These representations could in effect correspond to the rules prominent in several models of prefrontal function, linking information regarding context and incentive value with actions. This proposal predicts that behaviors

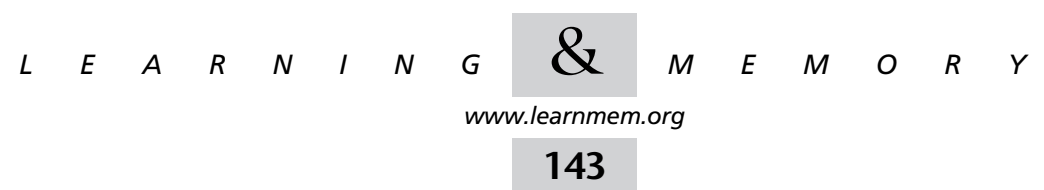



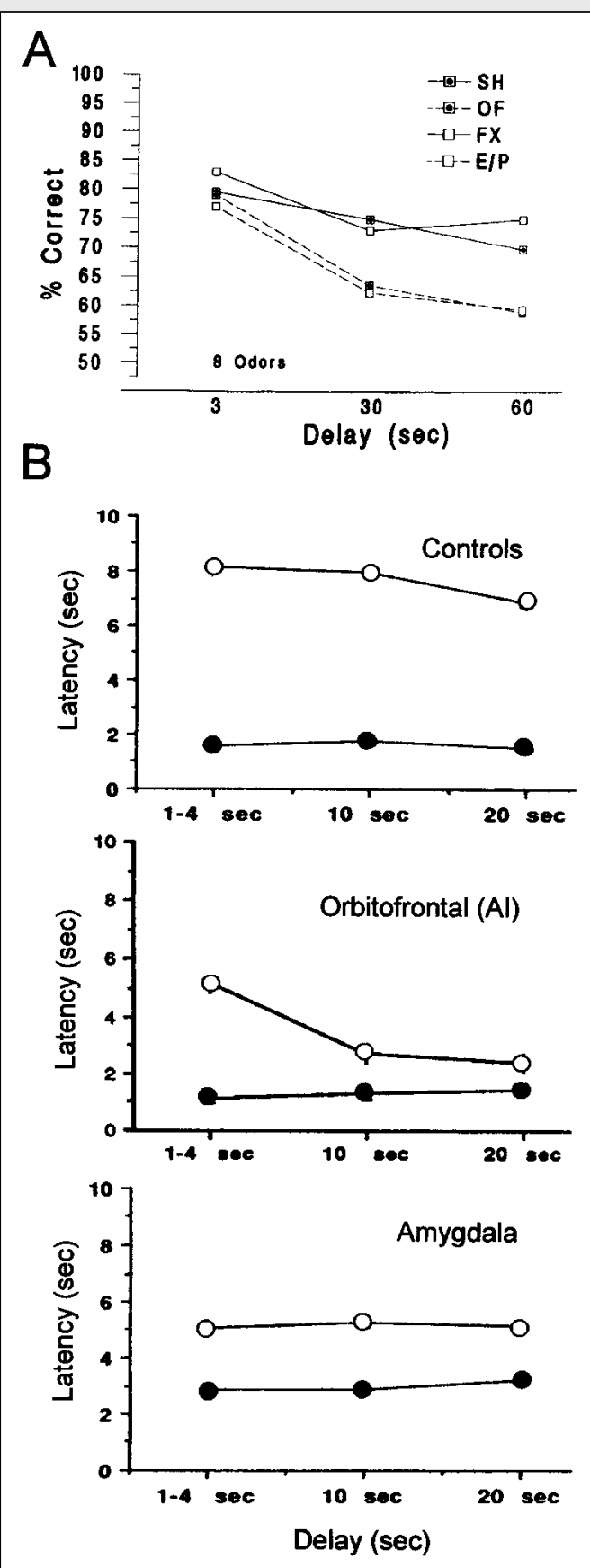

\section{Box 2. Delay-Dependent Deficits in Animals with Orbitofrontal Cortex Damage}

Animals with orbitofrontal lesions do occasionally show delay-dependent deficits that resemble those encountered after dorsolateral damage. For example, lesions of rat orbitofrontal cortex result in a delay-dependent deficit in performance of a continuous delayednonmatching to sample task (Otto and Eichenbaum 1992). In this task, an odor is presented on each trial and the rat must decide whether it matches the odor presented on the prior trial. If the odor does not match that presented on the prior trial, then a response will be rewarded; if the odor does match that presented on the prior trial, then a response will be unrewarded. The intertrial intervals are lengthened to create a delay. Both lesioned and control rats learn to inhibit responding to matching odors at very short delays when the intertrial interval is 0 or $3 \mathrm{sec}$. However, orbitofrontal cortex-lesioned rats exhibit worse performance at longer delays of 30 or $60 \mathrm{sec}$. The deficit caused by orbitofrontal cortex lesions in this task is indistinguishable from that of rats with perirhinal-entorhinal cortex aspiration lesions when as many as eight different odors are used (Fig. B2A). In addition, rats with orbitofrontal lesions restricted to agranular insular cortex exhibit delaydependent impairments on a magnitude-of-reinforcement discrimination task in which rats discriminate between objects paired with differing quantities of reward (Fig. B2B; DeCoteau et al. 1997). Delay-dependent deficits have also been reported in primates following damage to the orbitofrontal cortex (Mishkin 1964; Mishkin et al. 1969; Kowalska et al. 1991).

Such delay-dependent deficits following orbitofrontal cortex lesions have often been attributed to interitem interference and impaired inhibitory control rather than as a working memory deficit. This effect would seem to be particularly pronounced in a task such as delayed alternation or matching tasks, where the rewarded items change from trial to trial and the potential for interference is high. In this context, the well-documented discrimination and reversal deficit would perhaps account for the impairments, particularly when only two items are utilized. However, neurophysiological evidence suggests that there might be a working memory component of processing in the orbitofrontal cortex that would be important to performance in tasks stressing informational domains, such as incentive value, that are well represented in the orbitofrontal cortex. The results from the magnitude-of-reinforcement study are particularly noteworthy in this regard because they involve motivational information (magnitude of reinforcement) within a task in which, unlike alternation or delayed-nonmatch, the associations between particular items and reward do not change. Notably, the delay-dependent effect is specific to the orbitofrontal cortex; lesions of the amygdala (Fig. B2B) impair performance on this task but do not produce a delay-dependent deficit (Kesner and Williams 1995), and lesions in other prefrontal regions do not cause impairment (DeCoteau et al. 1997).

Figure B2 Effect of orbitofrontal lesions on delay tasks. (A) Effect of orbitofrontal lesions on performance in an eight-odor version of a continuous delayed-nonmatch to sample task. Rats were trained using 16 different odors, and performance was assessed using eight odors at delays (intertrial intervals) of 3, 30, and $60 \mathrm{sec}$. Testing was performed in rats with aspiration lesions of the orbitofrontal cortex (OF) or the perirhinal-entorhinal regions $(\mathrm{E} / \mathrm{P})$ or fornix transection $(\mathrm{FX})$, and results were compared to rats that received sham lesions $(\mathrm{SH})$. No impairments were observed at $3 \mathrm{sec}$, but both the orbitofrontal and perirhinal-entorhinal groups were impaired at longer delays. (Otto and Eichenbaum 1992, copyright 1992 by the American Psychological Association; reprinted with permission.) (B) Effect of orbitofrontal or amygdala lesions on performance on a magnitude-of-reinforcement discrimination task. In this task, the discriminative cues are cereal items for which rats exhibit different preferences. On each trial, rats received one of the two cereals during a study phase. After a study phase, the rats were allowed access into an adjoining area, and latency to displace an object to receive a hidden reward was assessed. The study phase could be lengthened after consumption of the cereal to create a delay. Intact rats displaced the object on positive trials (filled circles) faster than on negative trials (open circles) across all delays (top panel). Rats with bilateral electrolytic lesions of the agranular insular cortex showed a significant difference in the latency to displace the object on positive and negative trials at the short delays but not at longer delays (middle panel). Rats with bilateral electrolytic lesions of the amygdala showed no difference in the latency to displace the object at either short or long delays (bottom panel). (Kesner and Williams 1995, reprinted with permission from Academic Press; DeCoteau et al. 1997, reprinted with permission from Elsevier Science, see References for details.)

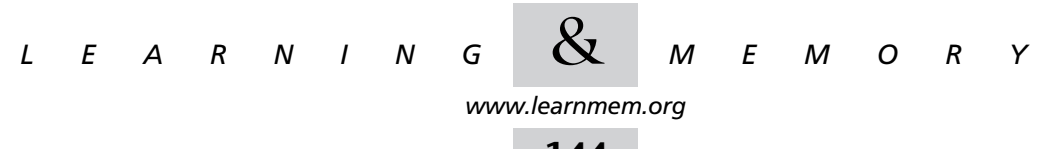


based on information critically contained in these representations, such as the incentive value of cues in a given context, will be disrupted by manipulations that alter these representations in intact animals.

The second of these features, implied by this model, is a working memory function, which allows information about cues to be accessed and manipulated even when those cues are not present in the environment. Networks in the orbitofrontal cortex would interact with other structures in this function in the same way that dorsolateral regions are proposed to function in concert with posterior cortical areas (Wilson et al. 1993; Goldman-Rakic 1996). Our discussion has highlighted encoding of incentive value and connections with the amygdala (Schoenbaum et al. 1998, 1999; Gallagher et al. 1999), but connections with other structures are also evident in the encoding properties of orbitofrontal neurons (Schoenbaum and Eichenbaum 1995a; Schoenbaum 1998; Lipton et al. 1999; Ramus and Eichenbaum 2000) and in activations reported in recent imaging studies (Frey and Petrides 2000). We predict that encoding in the orbitofrontal cortex should be sensitive to damage to related structures, and preliminary results indicate that this is indeed the case (Schoenbaum et al. 2001). In addition, performance across delays based on information critically represented in the orbitofrontal cortex, such as that regarding the incentive value of anticipated outcomes, should be adversely affected by orbitofrontal lesions.

In conclusion, effective rule learning seems to require representational memory capabilities, and representational memory divorced from the ability to acquire rules fails to describe the complex deficits attributable to damage to the prefrontal cortex. Accordingly, it appears that both rule learning and representational memory may provide the functional homogeneity proposed by Rosenkilde (1979) for this otherwise heterogeneous brain region.

\section{ACKNOWLEDGMENTS}

The authors thank Michela Gallagher for her support, Seth Ramus for his input, and Andrea Chiba and Don Katz for critical readings. This work was supported by K08-AG00882 to G.S. and F32MH12699 to B.S.

The publication costs of this article were defrayed in part by payment of page charges. This article must therefore be hereby marked "advertisement" in accordance with 18 USC section 1734 solely to indicate this fact.

\section{REFERENCES}

Adolphs, R., Bechara, A., Kaufman, O., Kawasaki, H., Bakken, H., Damasio, H., Granner, M., and Howard III, M. 2000. Single-unit responses in human orbitofrontal cortex: Decision-making on a gambling task. Seventh Ann. Meeting Cog. Neurosci. Soc., MIT Press, San Francisco.

Asaad, W.F., Rainer, G., and Miller, E.K. 2000. Task-specific neural activity in the primate prefrontal cortex. J. Neurophysiol. 84: 451-459.
Baxter, M.G., Parker, A., Lindner, C.C.C., Izquierdo, A.D., and Murray, E.A. 2000. Control of response selection by reinforcer value requires interaction of amygdala and orbitofrontal cortex. J. Neurosci. 20: 4311-4319.

Bechara, A., Damasio, H., Tranel, D., and Damasio, A.R. 1997. Deciding advantageously before knowing the advantageous strategy. Science 275: 1293-1294.

Bechara, A., Damasio, H., Tranel, D., and Andersen, S.W. 1998. Dissociation of working memory from decision making within the human prefrontal cortex. J. Neurosci. 18: 428-437.

Bechara, A., Damasio, H., Damasio, A.R., and Lee, G.P. 1999. Different contributions of the human amygdala and ventromedial prefrontal cortex to decision-making. J. Neurosci. 19: 5473-5481.

Butter, C.M., Mishkin, M., and Rosvold, H.E. 1963. Conditioning and extinction of a food-rewarded response after selective ablations of frontal cortex in rhesus monkeys. Exp. Neurol. 7: 65-75.

Cohen, J.D., Braver, T.S., and O'Reilly, R.C. 1998. A computational approach to prefrontal cortex, cognitive control, and schizophrenia: Recent developments and current challenges. In The prefrontal cortex (eds. A.C. Roberts et al.), pp. 195-220. Oxford University Press, New York.

Critchley, H.D. and Rolls, E.T. 1996a. Olfactory neuronal responses in the primate orbitofrontal cortex: Analysis in an olfactory discrimination task. J. Neurophysiol. 75: 1659-1672.

- 1996b. Hunger and satiety modify the responses of olfactory and visual neurons in the primate orbitofrontal cortex. J. Neurophysiol. 75: 1673-1686.

Damasio, A.R. 1994. Descartes' error. Putnam, New York.

DeCoteau, W.E., Kesner, R.P., and Williams, J.M. 1997. Short-term memory for food reward magnitude: The role of the prefrontal cortex. Behav. Brain Res. 88: 239-249.

Dias, R., Robbins, T.W., and Roberts, A.C. 1996. Dissociation in prefrontal cortex of affective and attentional shifts. Nature 380: 69-72.

Eichenbaum, H., Clegg, R.A., and Feeley, A. 1983. Reexamination of functional subdivisions of the rodent prefrontal cortex. Exp. Neurol. 79: 434-451.

Elliott, R., Friston, K.J., and Dolan, R.J. 2000. Dissociable neural responses in human reward systems. J. Neurosci. 20: 6159-6165.

Frey, S. and Petrides, M. 2000. Orbitofrontal cortex: A key prefrontal region for encoding information. Proc. Natl. Acad. Sci. 97: 8723-8727.

Funahashi, S., Bruce, C.J., and Goldman-Rakic, P.S. 1989. Mnemonic coding of visual space in the monkey's dorsolateral prefrontal cortex. J. Neurophysiol. 61: 331-349.

Fuster, J.M. 1985. The prefrontal cortex and temporal integration. In Cerebral cortex (eds. E.G. Jones and A. Peters), pp. 151-177. Plenum, New York.

Fuster, J.M., Bauer, R.H., and Jervey, J.P. 1982. Cellular discharge in the dorsolateral prefrontal cortex of the monkey in cognitive tasks. Exp. Neurol. 77: 679-694.

Gaffan, D. and Murray, E.A. 1990. Amygdalar interaction with the mediodorsal nucleus of the thalamus and the ventromedial prefrontal cortex in stimulus-reward associative learning in the monkey. $J$. Neurosci. 10: 3479-3493.

Gallagher, M. and Holland, P. 1999. Amygdala circuitry in attentional and representational processes. Trends Cog. Sci. 3: 65-73.

Gallagher, M., McMahan, R.W., and Schoenbaum, G. 1999. Orbitofrontal cortex and representations of incentive value in associative learning. $J$. Neurosci. 19: 6610-6614.

Gochin, P.M., Colombo, M., Dorfman, G.A., Gerstein, G.L., and Gross, C.G. 1994. Neural ensemble coding in inferior temporal cortex. $J$. Neurophysiol. 71: 2325-2337.

Goldman-Rakic, P.S. 1987. Circuitry of primate prefrontal cortex and regulation of behavior by representational memory. In Handbook of physiology: The nervous system (eds. F. Plum and V.B. Mountcastle), Vol. 5, pp. 373-417. The American Physiological Society, Bethesda, MD.

- 1996. The prefrontal landscape: Implications of functional

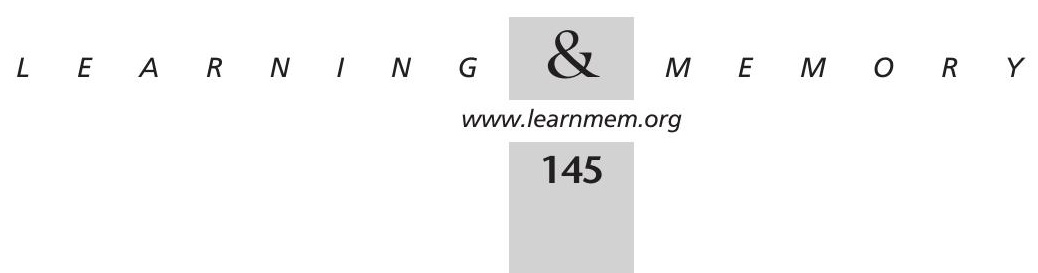


architecture for understanding human mentation and the central executive. Philos. Trans. Roy. Soc. Lond. [Biol.] 341: 1445-1453.

Harlow, J.M. 1868. Passage of an iron bar through the head. Pub. Mass. Med. Soc. 2: 329-346.

Hatfield, T., Han, J.-S., Conley, M., Gallagher, M., and Holland, P. 1996. Neurotoxic lesions of basolateral, but not central, amygdala interfere with Pavlovian second-order conditioning and reinforcer devaluation effects. J. Neurosci. 16: 5256-5265.

Hikosaka, K. and Watanabe, M. 2000. Delay activity of orbital and lateral prefrontal neurons of the monkey varying with different rewards. Cerebral Cortex 10: 263-271.

Holland, P.C. and Straub, J.J. 1979. Differential effects of two ways of devaluing the unconditioned stimulus after Pavlovian appetitive conditioning. J. Exp. Psychol.: Anim. Behav. Proc. 5: 65-78.

Izquierdo, A.D. and Murray, E.A. 2000. Bilateral orbital prefrontal cortex lesions disrupt reinforcer devaluation effects in rhesus. Soc. Neurosci. Abs. 26: 978 .

Jones, B. and Mishkin, M. 1972. Limbic lesions and the problem of stimulus-reinforcement associations. Exp. Neurol. 36: 362-377.

Kawasaki, H., Adolphs, R., Kaufman, O., Damasio, H., Damasio, A.R., Granner, M., Bakken, H., Hori, T., and Howard, M.A. 2001. Single-neuron responses to emotional visual stimuli recorded in human ventral prefrontal cortex. Nat. Neurosci. 4: 15-16

Kesner, R.P. 2000. Subregional analysis of mnemonic functions of the prefrontal cortex in the rat. Psychobiol. 28: 219-228.

Kesner, R.P. and Williams, J.M. 1995. Memory for magnitude of reinforcement: Dissociation between amygdala and hippocampus. Neurobiol. Learn. Memory 64: 237-244.

Kita, H. and Kitai, S.T. 1990. Amygdaloid projections to the frontal cortex and the striatum in the rat. J. Comp. Neurol. 298: 40-49.

Kowalska, D.M., Bechevalier, J., and Mishkin, M. 1991. The role of the inferior prefrontal convexity in performance of delayed nonmatching-to-sample. Neuropsychol. 29: 583-600.

Krettek, J.E. and Price, J.L. 1977. Projections from the amygdaloid complex to the cerebral cortex and thalamus in the rat and cat. $J$. Comp. Neurol. 172: 225-254.

Lipton, P.A., Alvarez, P., and Eichenbaum, H. 1999. Crossmodal associative memory representations in rodent orbitofrontal cortex. Neuron 22: 349-359.

Malkova, L., Gaffan, D., and Murray, E.A. 1997. Excitotoxic lesions of the amygdala fail to produce impairment in visual learning for auditory secondary reinforcement but interfere with reinforcer devaluation effects in rhesus monkeys. J. Neurosci. 17: 6011-6020.

McDonald, A.J. 1991. Organization of the amygdaloid projections to the prefrontal cortex and associated striatum in the rat. Neurosci. 44: 1-14.

McDonald, A.J., Mascagni, F., and Guo, L. 1996. Projections of the medial and lateral prefrontal cortices to the amygdala: A Phaseolus vulgaris leucoagglutinin study in the rat. Neurosci. 71: 55-75.

Meunier, M., Bachevalier, J., and Mishkin, M. 1997. Effects of orbital frontal and anterior cingulated lesions on object and spatial memory in rhesus monkeys. Neuropsychol. 35: 999-1015.

Miller, E.K. 2000. The prefrontal cortex and cognitive control. Nature Rev. Neurosci. 1: 59-65.

Mishkin, M. 1964. Preservation of central sets after frontal lesions in monkeys. In The frontal granular cortex and behavior (eds. J.M Warren and K. Akert), pp. 219-241. McGraw-Hill, New York.

Mishkin, M., Vest, B., Waxler, M., and Rosvold, H.E. 1969. A reexamination of the effects of frontal lesions on object alternation. Neuropsychol. 7: 357-363.

Nobre, A.C., Coull, J.T., Frith, C.D., and Mesulam, M.M. 1999. Orbitofrontal cortex is activated during breaches of expectation in tasks of visual attention. Nature Neurosci. 2: 11-12.

O'Doherty, J., Kringelbach, M.L., Rolls, E.T., Hornak, J., and Andrews, C. 2001. Abstract reward and punishment representations in the human orbitofrontal cortex. Nature Neurosci. 4: 95-102.
Ongur, D. and Price, J.L. 2000. The organization of networks within the orbital and medial prefrontal cortex of rats, monkeys and humans. Cerebral Cortex 10: 206-219.

Onoda, N., Imamura, K., Obota, F., and Iino, M. 1984. Responses selectivity of neocortical neurons to specific odors in the rabbit. $J$. Neurophysiol. 52: 638-652.

Otto, T. and Eichenbaum, H. 1992. Complementary roles of the orbital prefrontal cortex and the perirhinal-entorhinal cortices in an odor-guided delayed-nonmatching-to-sample task. Behav. Neurosci. 106: $762-775$.

Passingham, R. 1993. The frontal lobes and voluntary action. Oxford University Press, New York.

Petrides, M. 1994. Frontal lobes and working memory: Evidence from investigations of the effects of cortical excisions in nonhuman primates. In Handbook of neuropsychology (eds. F. Boller and J. Grafman), Vol 9, pp. 59-82. Elsevier, Amsterdam.

Preuss, T.M. 1995. Do rats have prefrontal cortex? The Rose-WoolseyAkert program reconsidered. J. Cog. Neurosci. 7: 1-24.

Price, J.L., Russchen, F.T., and Amaral, D.G. 1987. The limbic region. II The amygdaloid complex. In Integrated systems of the CNS. Part I. Handbook of chemical neuroanatomy (eds. A. Bjorklund et al.), Vol. 5, pp. 279-388. Elsevier, Amsterdam.

Rainer, G., Asaad, W.F., and Miller, E.K. 1998. Selective representation of relevant information by neurons in the primate prefrontal cortex. Nature 393: 577-579.

Ramus, S.J. and Eichenbaum, H. 2000. Neural correlates of olfactory recognition memory in the rat orbitofrontal cortex. J. Neurosci. 20: 8199-8208.

Robbins, T.W. 1996. Dissociating executive functions of the prefrontal cortex. Philos. Trans. Roy. Soc. Lond. [Biol.] 351: 1463-1471.

Rogers, R.D., Owen, A.M., Middleton, H.C., Williams, E.J., Pickard, J.D., Sahakian, B.J., and Robbins, T.W. 1999. Choosing between small, likely rewards and large, unlikely rewards activates inferior and orbital prefrontal cortex. J. Neurosci. 20: 9029-9038.

Rolls, E.T., Hornak, J., Wade, D., and McGrath, J. 1994. Emotion-related learning in patients with social and emotional changes associated with frontal lobe damage. J. Neurol. Neurosurg. Psychiatry 57: 1518-1524

Rolls, E.T., Critchley, H.D., Mason, R., and Wakeman, E.A. 1996. Orbitofrontal cortex neurons: Role in olfactory and visual association learning. J. Neurophysiol. 75: 1970-1981.

Rosenkilde, C.E. 1979. Functional heterogeneity of the prefrontal cortex in the monkey: A review. Behav. Neural. Biol. 25: 301-345.

Rosenkilde, C.E., Bauer, R.H., and Fuster, J.M. 1981. Single cell activity in ventral prefrontal cortex in behaving monkeys. Brain Res. 209: 375-394.

Saddoris, M.P., Setlow, B., Nugent, S., and Schoenbaum, G. 2000. A reexamination of the role of orbitofrontal cortex and basolateral amygdala in acquisition and reversal of odor-guided go, no-go discrimination task. Soc. Neurosci. Abs. (in press)

Scannell, J.W. and Young, M.P. 1999. Neuronal population activity and functional imaging. Proc. Roy. Lond. Soc. B 266: 875-881.

Schoenbaum, G. 1998. Cell assemblies and the ghost in the machine. In Neuronal ensembles: Strategies for recording and decoding (eds. H. Eichenbaum and J.L. Davis), pp. 81-116. Wiley-Liss, New York.

Schoenbaum, G. and Eichenbaum, H. 1995a. Information coding in the rodent prefrontal cortex. I. Single neuron activity in orbitofrontal cortex compared with that in piriform cortex. J. Neurophysiol. 74: 733-750.

- $1995 \mathrm{~b}$. Information coding in the rodent prefrontal cortex. II. Ensemble activity in orbitofrontal cortex. J. Neurophysiol. 74: 751-762.

Schoenbaum, G., Chiba, A.A., and Gallagher, M. 1998. Orbitofrontal cortex and basolateral amygdala encode expected outcomes during learning. Nature Neurosci. 1: 155-159.

1999. Neural encoding in orbitofrontal cortex and basolateral

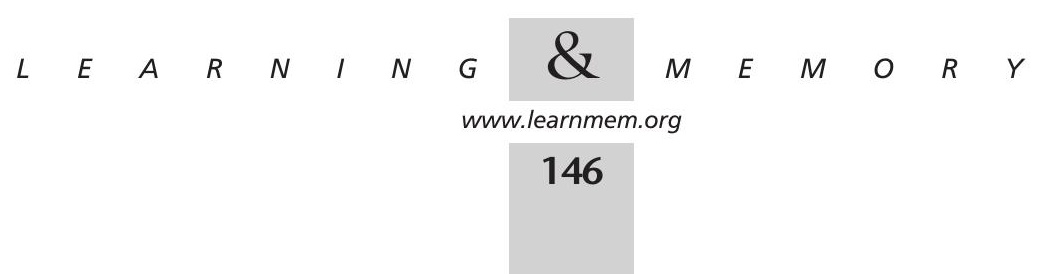


amygdala during olfactory discrimination learning. J. Neurosci. 19: $1876-1884$.

. 2000. Changes in functional connectivity in orbitofrontal cortex and basolateral amygdala during learning and reversal training. $J$. Neurosci. 20: 5179-5189.

Schoenbaum, G., Setlow, B., Saddoris, M.P., and Gallagher, M. 2001. Basolateral amygdala lesions disrupt neural correlates of incentive value in orbitofrontal cortex. Soc. Neurosci. Abs. (in press).

Schoenbaum, G., Setlow, B., and Gallagher, M. Orbitofrontal cortex: Modeling prefrontal function in rats. In The neuropsychology of memory, 3rd ed. (eds. L. Squire and D. Schacter) (in press)

Schultz, W., Tremblay, L., and Hollerman, J.R. 2000. Reward processing in primate orbitofrontal cortex and basal ganglia. Cerebral Cortex 10: $272-283$.

Tanabe, T., Ino, M., and Takagi, S.F. 1975. Discrimination of odors in olfactory bulb, pyriform-amygdaloid areas, and orbitofrontal cortex of the monkey. J. Neurophysiol. 38: 1284-1296.

Thorpe, S.J., Rolls, E.T., and Maddison, S. 1983. The orbitofrontal cortex: Neuronal activity in the behaving monkey. Exp. Brain Res. 49: 93-115.

Tremblay, L. and Schultz, W. 1999. Relative reward preference in primate orbitofrontal cortex. Nature 398: 704-708.

\footnotetext{
- 2000a. Reward-related neuronal activity during go-no go task performance in primate orbitofrontal cortex. J. Neurophysiol. 83: $1864-1876$.

-2000b. Modifications of reward expectation-related neuronal activity during learning in primate orbitofrontal cortex. $J$. Neurophysiol. 83: 1877-1885.

Watanabe, M. 1996. Reward expectancy in primate prefrontal neurons. Nature 382: 629-632.

Wilson, F.A.W., Scalaidhe, S.P.O., and Goldman-Rakic, P.S. 1993. Dissociation of object and spatial processing domains in primate prefrontal cortex. Science 260: 1955-1958.

Wise, S.P. and Murray, E.A. 2000. Arbitrary associations between antecedents and actions. Trends Neurosci. 23: 271-276.

Wise, S.P., Murray, E.A., and Gerfen, C.R. 1996. The frontal cortex-basal ganglia system in primates. Crit. Rev. Neurobiol. 10: 317-356.

Yonemori, M., Nishijo, H., Uwano, T., Tamura, R., Furita, I., Kawasaki, M. Takashima, Y., and Ono, T. 2000. Orbital cortex neuronal responses during an odor-based conditioned associative task in rats. Neurosci. 95: 691-703.
}

Received February 26, 2001; accepted in revised form April 16, 2001. 


\section{Erratum}

Learning \& Memory 8: 134-147 (2001)

Integrating Orbitofrontal Cortex into Prefrontal Theory: Common Processing Themes across Species and Subdivisions

Geoffrey Schoenbaum and Barry Setlow

The title for the Saddoris, M.P., Setlow, B., Nugent, S., and Schoenbaum, G. reference should correctly read: A reexamination of the role of orbitofrontal cortex in acquisition and reversal of odor-guided go, no-go discriminations. In addition, the year of publication was incorrectly shown as 2000, rather than as 2001 (in press).

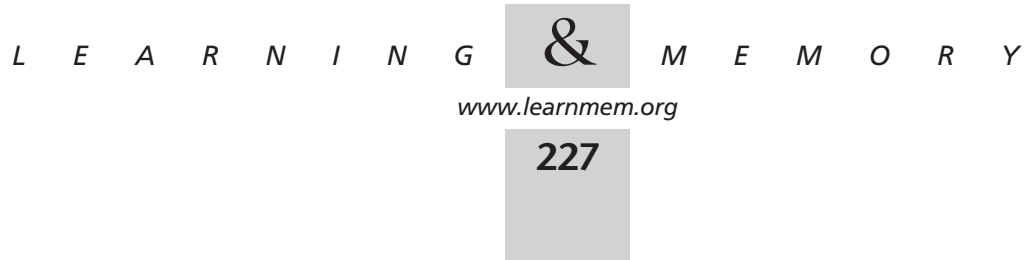




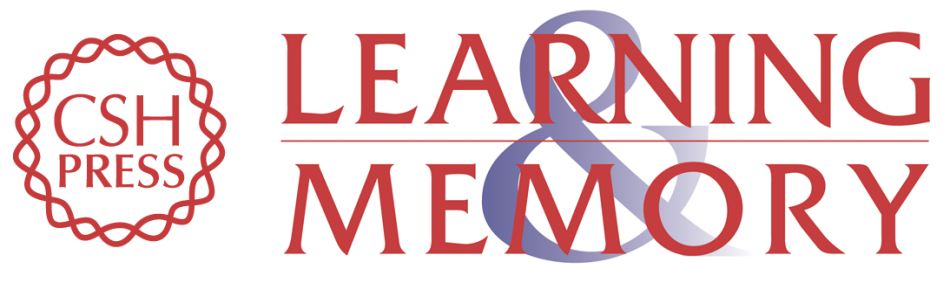

\section{Integrating Orbitofrontal Cortex into Prefrontal Theory: Common Processing Themes across Species and Subdivisions}

Geoffrey Schoenbaum and Barry Setlow

Learn. Mem. 2001, 8:

Access the most recent version at doi:10.1101//m.39901

\section{Related Content Errata for vol. 8, p. 134}

Learn. Mem. July, 2001 8: 227

References This article cites 67 articles, 15 of which can be accessed free at: http://learnmem.cshlp.org/content/8/3/134.full.html\#ref-list-1

Articles cited in:

http://learnmem.cshlp.org/content/8/3/134.full.html\#related-urls

License

Email Alerting Receive free email alerts when new articles cite this article - sign up in the box at the Service top right corner of the article or click here. 